\title{
Experience of Teaching Advanced Touch Sensing Technologies
}

\author{
Nannan He, Han-Way Huang \\ Department of Electrical, Computer Engineering and Technology \\ Minnesota State University, Mankato, MN 56001
}

\begin{abstract}
A touchscreen is an electronic visual display that users can control by touching the screen with a special stylus or fingers. It is popular in many information appliances such as tablet computers, smartphones, and personal digital assistants (PDAs). In fact, display manufactures and chip vendors around the world, such as Samsung, Chimei, Atmel, ST Microelectronics, and Texas Instruments, have recognized the trend of using touchscreens as a highly desirable user interface component and started to integrate the touch-sensing technology into their products. This paper presents our experience of teaching touch sensing technology in our advanced level microprocessor course. During the course our students have shown great interests in touch sensing technologies and are capable of incorporating touch devices to improve the human machine interface of their capstone projects.
\end{abstract}

\section{Introduction}

Touchscreens allow the rapid, accurate and direct interaction by the user with display contents, which existing keyboard and mouse systems cannot. There are many touch sensing technologies. Among them, analog resistive, surface capacitive, projected capacitive, infrared grid, optical imaging, and surface acoustic wave are the most important ones. We feel the importance and need to teach engineering students the touch sensing technology. This paper presents our experience of teaching touch sensing technology to engineering and technology students. We taught the technology in slightly over 5 weeks. As the start of the course, we introduced working principles of each touch technology to students. We then conducted a comparison among these technologies and their applications in real-world electronic devices. Students were taught to program a touchscreen through a series of lab exercises. The Atmel SAM 4S-EK board was the main development board employed in the course for practicing touchscreen programming. This board includes four QTouch buttons and slides which utilize capacitive sensing technology, and a resistive touch panel on a color LCD display. Atmel provides a royalty free software library for developing touch applications in C. Students learned to link the library into their applications so as to provide touch sensing capability in their projects. In this paper, we first introduce the history of touch screen technology and five common touch sensing technologies. Then we present capstone projects developed by technology students using the Atmel 4S-EK board which involve QTouch devices. Finally, the paper is ended with conclusions and future work. 


\section{A Brief History of Touch Screen Technology}

In 1967, E.A. Johnson at the Royal Radar Establishment, UK published his work in an article on the capacitive touch screen, which is widely used in smart phones. It is believed by most the world's first touch screen. In 1974, Dr. Hurst with his company Elographics produced the first touchscreen featuring a transparent surface. And the following year, Elographics patented a resistive touch screen technology, one of the most popular touchscreen technologies in use today. In 1983, Hewlett-Packard introduced the HP-150, a home computer with touch screen technology. It had a built-in grid of infrared beams across the front of the monitor which detected finger movements. However, the infrared sensors would collect dust and require frequent cleanings. In 1990s, smart phones and handhelds with touch screen technology were introduced. In 1993, Apple released the Newton PDA, equipped with handwriting recognition; and IBM released the first smart phone called Simon, which featured a calendar, note pad, and fax function, and a touch screen interface that allowed users to dial phone numbers. In 1996, Palm entered the PDA market and advanced touch screen technology with its Pilot series. In 2002, Microsoft introduced the Windows XP Tablet edition and started its entry into touch technology. In 2007, Apple introduced the king of smart phones - iPhone with touch screen technology. Since then, the touch screen became wildly popular on handhelds.

\section{Touch Sensing Technologies}

A touch screen typically consists of three components:

- The touch sensor is a panel with a touch responsive surface. Systems are built based on different types of sensors: resistive (most common), surface acoustic wave, and capacitive (most smart phones). In general, sensors have an electrical current running through them and touching the screen causes a voltage change. The voltage change signals the location of the touching.

- The controller is the hardware that converts the voltage changes on the sensor into signals the computer or other device can recognize.

- The software interprets sensor signals and the information coming from the controller, and allows the computer or smart phone to react accordingly.

Five common touch sensing technologies: analog resistive, surface capacitive, projected capacitive are described in the following.

\section{Analog resistive touch sensing}

An analog resistive touchscreen typically consists of two layers as shown in Figure 1, a coversheet layer made of Polyethylene (PET) and a rigid back layer made of glass. Both layers are coated with an electrically conductive compound called Indium Tin Oxide (ITO). The 
coversheet has a flexible, durable coating on the outer side. These two layers are separated with tiny and transparent insulating spacer dots.

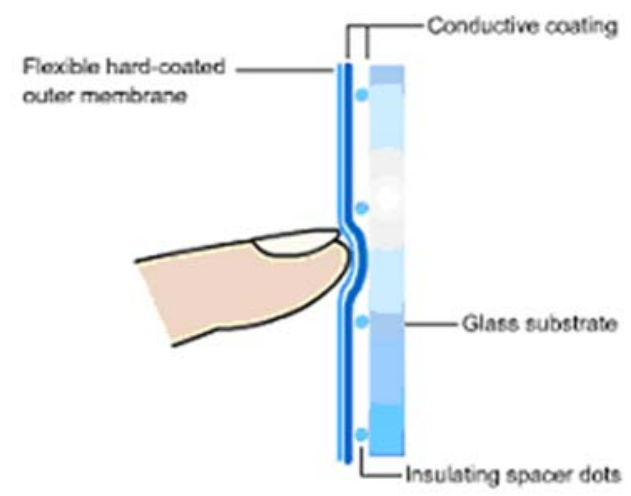

Figure 1. Illustration of Resistive Touch (Courtesy of Elo TouchSystems)

When the screen is touched, it pushes the conductive coating on the coversheet against the coating on the back glass, making electrical contact. The voltages produced are the analog representation of the position touched. An electronic controller converts these voltages into digital $\mathrm{X}$ and $\mathrm{Y}$ coordinates which are transmitted to the host computer for the software to find the relative position of the touch activation. The resistive touch technology has several implementation variations, such as the 4-, 5-, 6-, 7- and 8-wired touch screen. While these variants are constructed similarly, they are different in how they determine the coordinates of the touch ${ }^{1}$. Due to the space limit, we only describe the four-wire resistive touch screen.

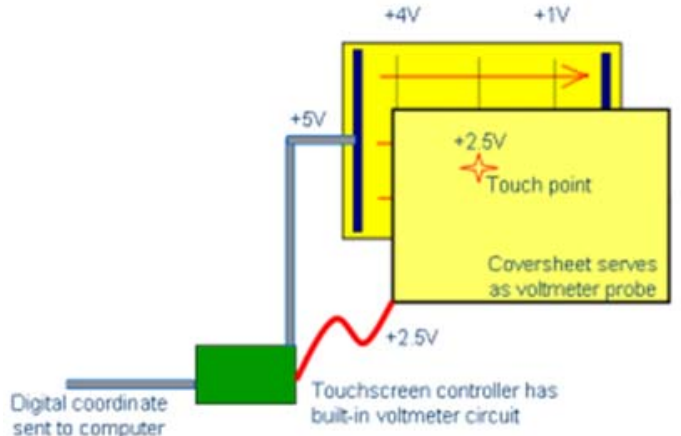

(a)

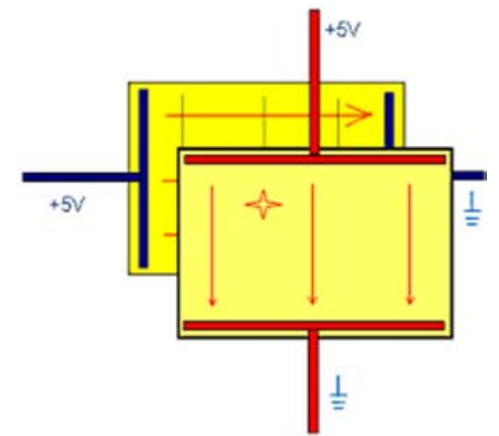

(b)

Figure 2 Resistive touchscreen

\section{$\underline{\text { Four-wire resistive touchscreen }}$}

Four-wire resistive technology is the simplest to manufacture. It is constructed with uniform coatings of ITO on the inner sides of the layers and silver bars (blue or red lines) along the edges. Such combination sets up lines of equal potential in both X and Y. It uses both the upper and lower layers in the touchscreen to determine the $\mathrm{X}$ and $\mathrm{Y}$ coordinates of a touch. Touch measurement in the four-wire is a two steps process. The $\mathrm{x}$-coordinate of the touch point is calculated by creating a voltage gradient on the flexible layer, and the $y$-coordinate is 
determined by creating a voltage gradient along the bottom layer. In the Figure 2(a), the controller first applies $5 \mathrm{~V}$ to the back layer. Upon touch, it probes the analog voltage with the coversheet, reading $2.5 \mathrm{~V}$, which represents a left-right position or $\mathrm{X}$ axis. It then flips the process as shown in Figure 2(b), applying 5V to the coversheet, and probes from the back layer to calculate an up-down position or $\mathrm{Y}$ axis. Four-wire resistive touchscreens are relatively cheap, easily available and consume low power. However, with this technology, one coordinate axis (usually the $\mathrm{Y}$ axis), uses the outer flexible coversheet as a uniform voltage gradient. The constant flexing that occurs on the coversheet will cause microscopic cracks in the ITO coating, changing its electrical characteristics (i.e., resistance), degrading the linearity and accuracy of this axis. Also, accuracy can drift with environmental changes. While these drawbacks can be insignificant in smaller sizes, they become increasingly apparent the larger the touchscreen. Therefore, four-wire touchscreens are normally recommended in applications with a small display size.

In summary, resistive touch sensors are cost effective to manufacture because they have simple structures and offer a high degree of accuracy. They are also the most widely used touch sensing technology. A disadvantage of resistive touch technology is shorter lifetimes due to mechanical wear and tear, which makes it not very suitable for harsh environments. And this technology is susceptible to temperature and moisture extremes.

\section{Capacitive touch sensing}

A capacitive touchscreen panel generally consists of an insulator such as glass, coated with a transparent conductive material, such as ITO to hold an electrical charge. The human body is an electrical conductor, so touching the screen with a finger distorts the screen's electrostatic field, which can be measured as a capacitance change at the location of the touch. The location is then sent to the controller for processing.

\section{$\underline{\text { Surface-Capacitive Touchscreen }}$}

This technology works on the basis of a person or conductive stylus creating a path for an electrical current to flow from the surface of a touch screen to ground. Specifically, the human body has inherent impedance ranging from about $20 \mathrm{k}$ to $300 \mathrm{k} \Omega$, which is much smaller than the inherent leakage impedance from the touch screen to ground. Electrodes around the panel's edge evenly distribute a low voltage across the conductive layer, resulting in a uniform

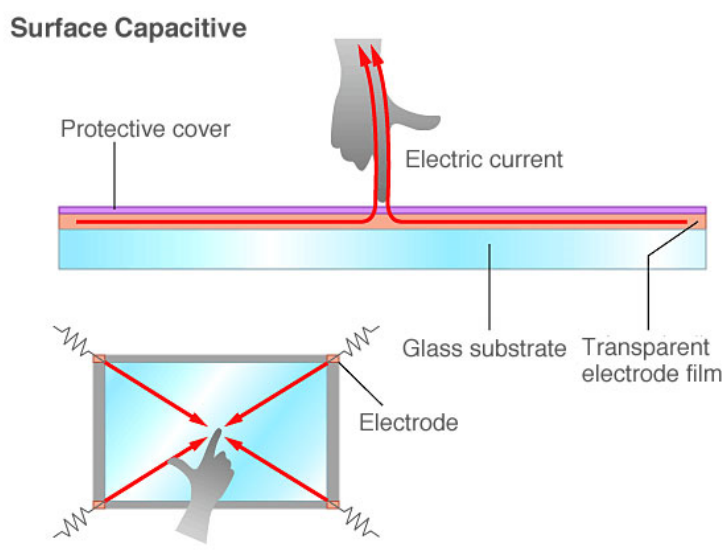

Figure 3 Surface Capacitive electrostatic field over the conductive layer. Whenever the screen is touched, the current is 
drawn from each corner and flows through the finger, through the person's body impedance, then through a body-to-ground impedance and back to the ground as shown in Figure 3 . The touch current flow is very small —in the range of 20 to $500 \mu \mathrm{A}$. This current exceeds the touch threshold set by the controller and the position of the touchdown location is precisely calculated as being inversely proportional to the distance from the contact point to the corner. As shown in Figure 4, surface capacitive touch sensors are made with one sheet of glass that is coated with a conductive coating and a linearization pattern. On top of these layers, it is applied with a protective hard coat with anti-glare properties. Some manufactures like $3 \mathrm{M}$ may add a scratch resistant top coat for additional protection against sharp objects scratching the screen.

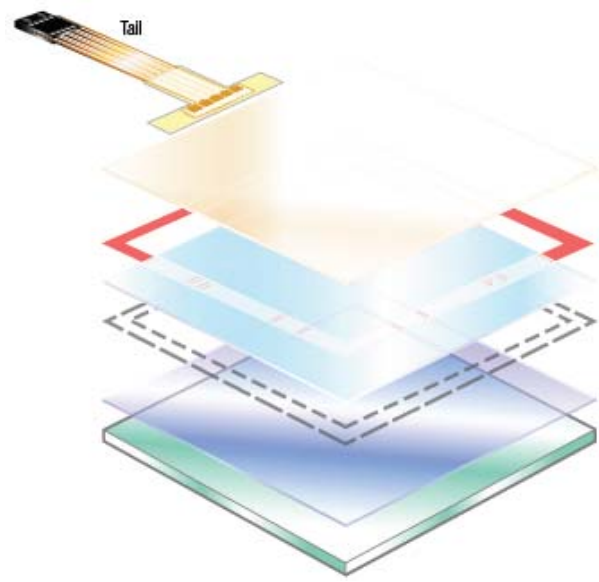

Figure 4 Capacitive Touch Manufacturing Layers (Courtesy of 3M)

This type of technology is commonly used within the gaming industry. Its function is unaffected by moisture, temperature, and dust. Since there is no flexing of the surface, the actuations are very high, capable of reaching over 100 million times, which implies better durability. The drawback of this technology is that it is only a single touch. It has low resolution, is prone to false signals from parasitic capacitive coupling, and needs calibration during manufacture. The user will also have to use a finger or conductive stylus. Overall, the surface capacitive touchscreen is moderately durable and needs calibration during manufacture. Since a conductive material is required to operate this screen, passive stylus cannot be used for surface capacitive touchscreen.

\section{Projected-Capacitive Touchscreen}

Projected Capacitive Technology (PCT) is fast becoming one of the most prevalent touch technologies for an expanding variety of applications ranging from consumer devices to commercial applications in retail, gaming and signage. PCT detects touch by measuring the capacitance at each addressable electrode. When a finger or a conductive stylus approaches an electrode, it disturbs the electromagnetic field and alters the capacitance. This change in 
capacitance can be measured by the electronics and then converted into X, Y locations that the system can use to detect touch. There are two main types of sensing methods, self-capacitance and mutual capacitance ${ }^{2}$. To sense touch in a self-capacitance implementation, the electronics scans through each electrode and measures the amount of current on each electrode to establish a steady-state current. When a touch approaches the screen, the electrons couple to the electrodes and increases the current as it creates a path to ground. As shown in Figure 5, the electronics measure the current on each electrode to ground in this implementation. This method produces a stronger signal than mutual capacitance, but it is unable to resolve accurately more than one finger, which results in misplaced location sensing.

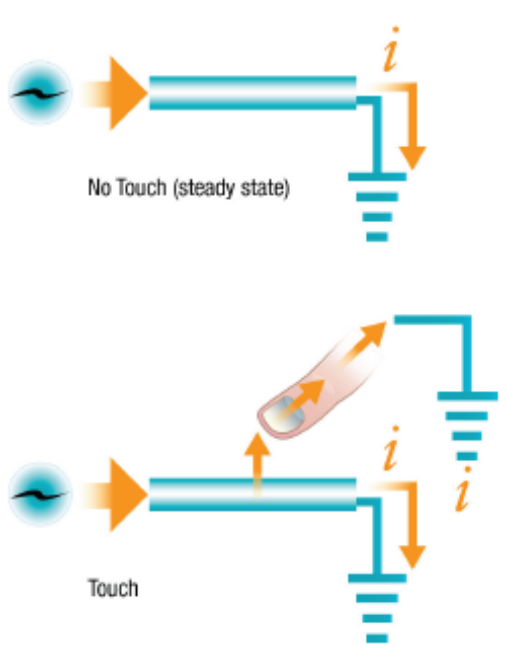

Figure 5 How Self Capacitance works (Courtesy of $3 \mathrm{M}$ )

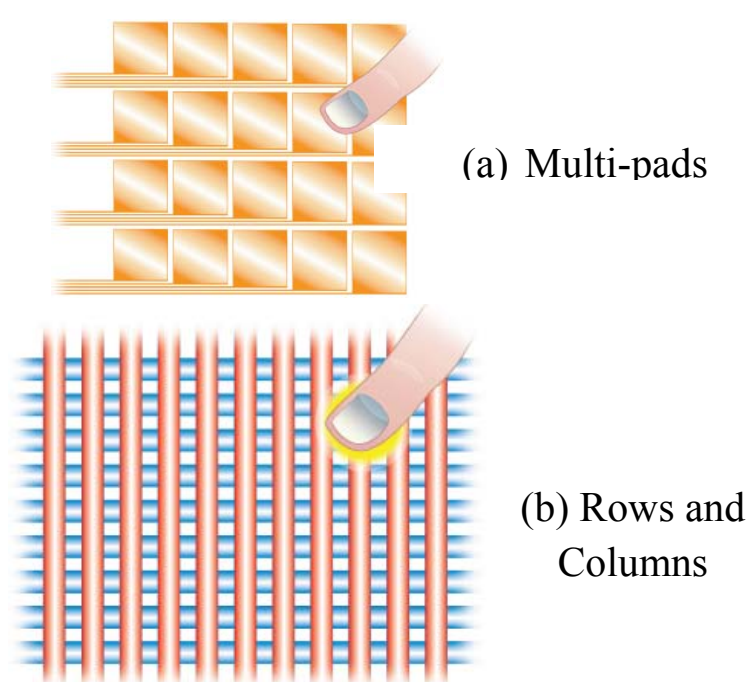

Figure 6 Self capacitance construction (Courtesy of $3 \mathrm{M})$

A touch can be detected in two different manners: multi-pad construction or rows and columns. In a multi-pad construction as in Figure 6 (a), each electrode, or "pad", is individually addressable by the electronics requiring an individual connection between the electrode and the controller. This allows multi-pad self-capacitance to support greater than one touch, but given that each pad must be individually addressed, it makes the implementation for screens greater than 3.5 inches very challenging. In a row-and-column construction as in Figure 6 (b), each row and column is an electrode and therefore is individually addressed by the controller. Even though the intersection of a row and column represents a unique coordination pair, the electronics cannot measure each individual intersection as they can only measure each electrode. This limits row and column self-capacitance implementations to single and dual touch detection where "ghost" points can be a problem.

Mutual capacitance is the capacitance between two "charge holding objects." Projected capacitance touchscreens intentionally create mutual capacitance between elements of columns and rows in the vicinity where each intersect the other as in Figure 7. This allows the system 
electronics to measure each node (intersection) individually to detect multiple touches on the screen during one screen scan.
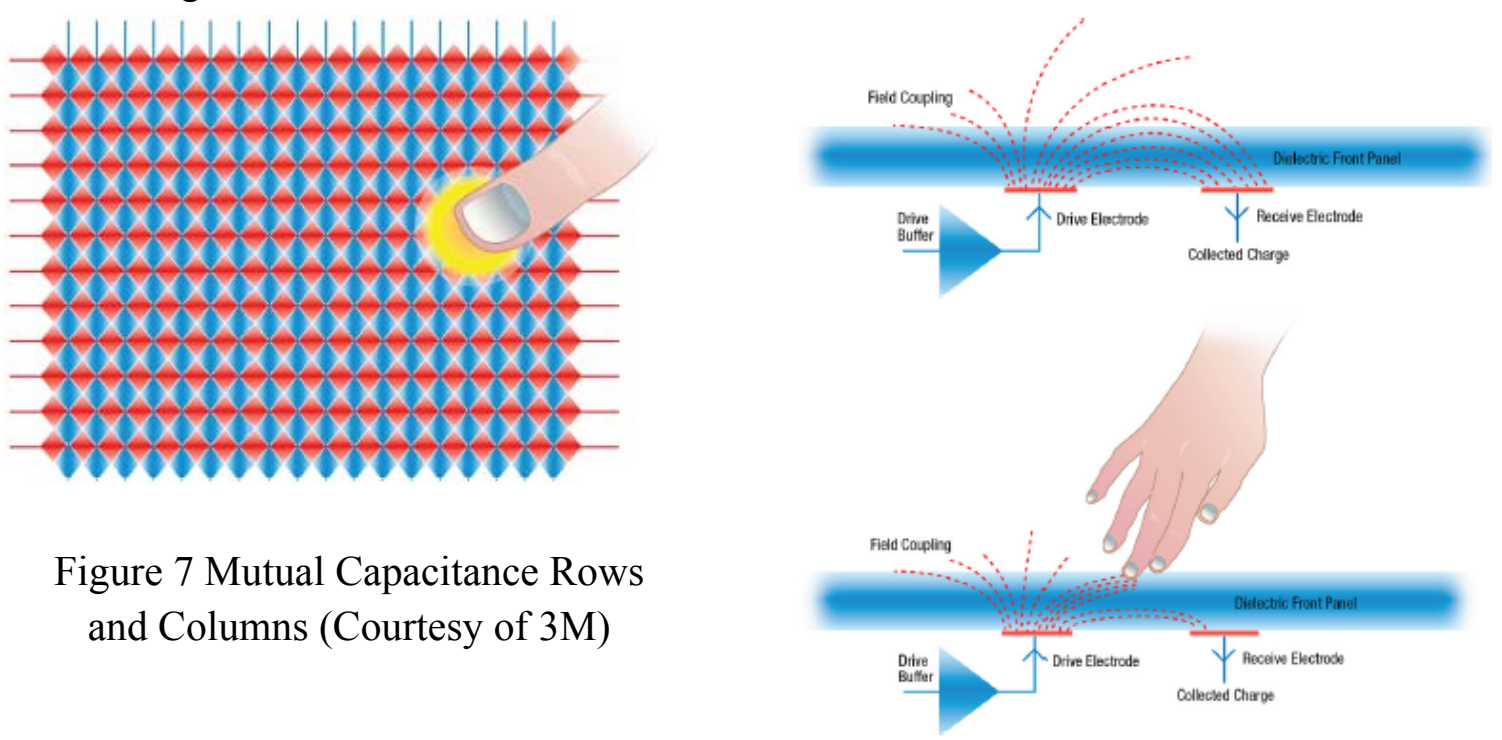

Figure 7 Mutual Capacitance Rows and Columns (Courtesy of 3M)

Figure 8 How Mutual Capacitance Works

When a finger touches near an intersection, as shown in Figure 8, some of the mutual capacitance between the row and column is coupled to the finger which reduces the capacitance at the intersection as measured by the system electronics. This reduced capacitance crosses the "touch threshold" set by the electronics indicating a touch has occurred.

\section{Touch Technologies Comparisons}

We compare common touch sensing technologies, and rank them ( 5 - best, 1 - worst, 0 - not applicable) based on a set of features as shown in the following table ${ }^{3}$.

\begin{tabular}{|l|l|l|l|l|l|}
\hline Features & $\begin{array}{l}\text { Resistive } \\
(4-w i r e)\end{array}$ & $\begin{array}{l}\text { Surface } \\
\text { Capacitive }\end{array}$ & $\begin{array}{l}\text { Projected } \\
\text { Capacitive }\end{array}$ & $\begin{array}{l}\text { Surface } \\
\text { acoustic } \\
\text { Wave }\end{array}$ & Infrared \\
\hline Ambient Light Sensitivity & 5 & 5 & 5 & 5 & 3 \\
\hline Calibration Stability & 2 & 2 & 5 & 4 & 5 \\
\hline Cost & 5 & 3 & 2 & 4 & 1 \\
\hline Durability & 1 & 4 & 5 & 4 & 5 \\
\hline Multi-Touch & 0 & 0 & 5 & 0 & 5 \\
\hline Weather Resistance & 2 & 3 & 5 & 2 & 2 \\
\hline Best applicable size & $2 "-26 "$ & $12 "-26 "$ & $2 "-10 "$ & 12 "-26" & 12 "-24" \\
\hline Mobile/Handheld & 5 & 0 & 5 & 0 & 0 \\
\hline
\end{tabular}

Table 1. Technologies Comparison 


\section{Experience of Teaching Touch Devices Programming}

We present our experience of teaching engineering and technology students the knowledge of Touch devices from three aspects: development board selection, touch device programming and cape stone projects.

\section{Touch elements on the SAM4S Evaluation Kit (SAM4S-EK)}

The computing capability of MCUs (for example, the ARM Cortex-A and Cortex-M series) increases dramatically. Almost all microcontroller vendors provide their touch technology solutions, which consist of Touch devices either embedded in some development boards or as individual hardware like a BoosterPack, software library to program the Touch hardware and other facilities to assist developing the touch-based projects. Nowadays, many low-cost development boards are equipped with Touch elements that can be used for the education purpose. For example, the STM32L1 discovery board from ST Microelectronics includes a linear touch sensor and four touch keys, the software library for programming such touch elements are also provided; the Freescale FRDM-KL25Z development platform provides a capacitive touch slider; Texas Instrument offers low power touch devices for buttons, sliders, wheels and proximity applications.

Atmel offered their Touch solutions based on two types of patented capacitive touch acquisition methods, QTouch and QMatrix. QTouch is based on the self-capacitance sensing technology and detects touches by measuring the charge flow at a given point. It is reported to have good proximity and be easy to tune sensitivity. In comparison, QMatrix detects touch using a scanned passive matrix of electrode sets. It basically belongs to the mutual-capacitance sensing technology and is resilient to moisture \& environment changes. The SAM4S-EK includes a Cortex-M4 processor, a 2.8" color LCD display with touch panel and backlight, and rich touch elements. The touch panel implements five capacitive QTouch keys (UP, DOWN, RIGHT, LEFT and VALID) using five pairs of IO pins. Each key needs one channel. The board also has a Slider which is formed by three channels for a QTouch acquisition method using three pairs of IO pins. It is used to detect a linear finger displacement on a sensitive area.

\section{Programming QTouch}

Atmel QTouch Library is a free software library for developing touch applications on standard AVR and SAM microcontrollers ${ }^{4}$. Customers can link the library into their applications in order to provide touch sensing capability in their projects as shown in Figure 9. It can be used to develop single chip solutions for control applications with touch sensing capabilities, or to develop standalone touch sensing solutions which interface with other host or control devices. 


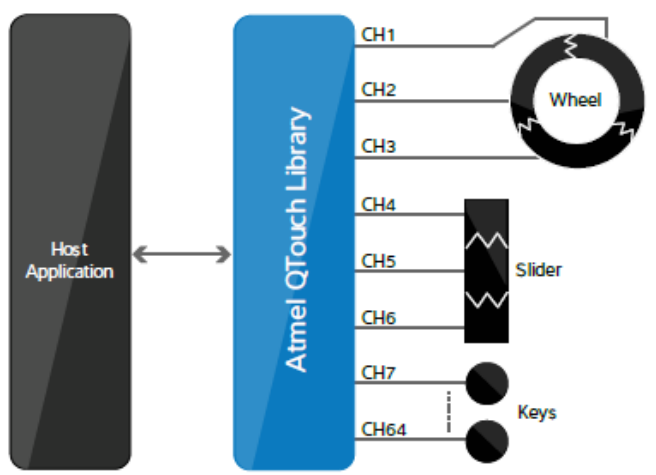

Figure 9 QTouch library in courtesy of Atmel

Using the API functions provided in the QTouch library, touch sensors and associated channels can be defined. Once touch sensing has been initiated by the user, the host application can use the API to make touch measurements and determine the status of the sensors. Atmel studio provides one example project "QTouch Method Example Application” for developing SAM4SEK applications. Students can make use of this project as the basis to start their project. They could learn how to include QTouch library into their application project, compile with other application code to generate executable files. The ASF wizard facilitates adding APIs in the QTouch Library via a service module as highlighted in Figure 10.

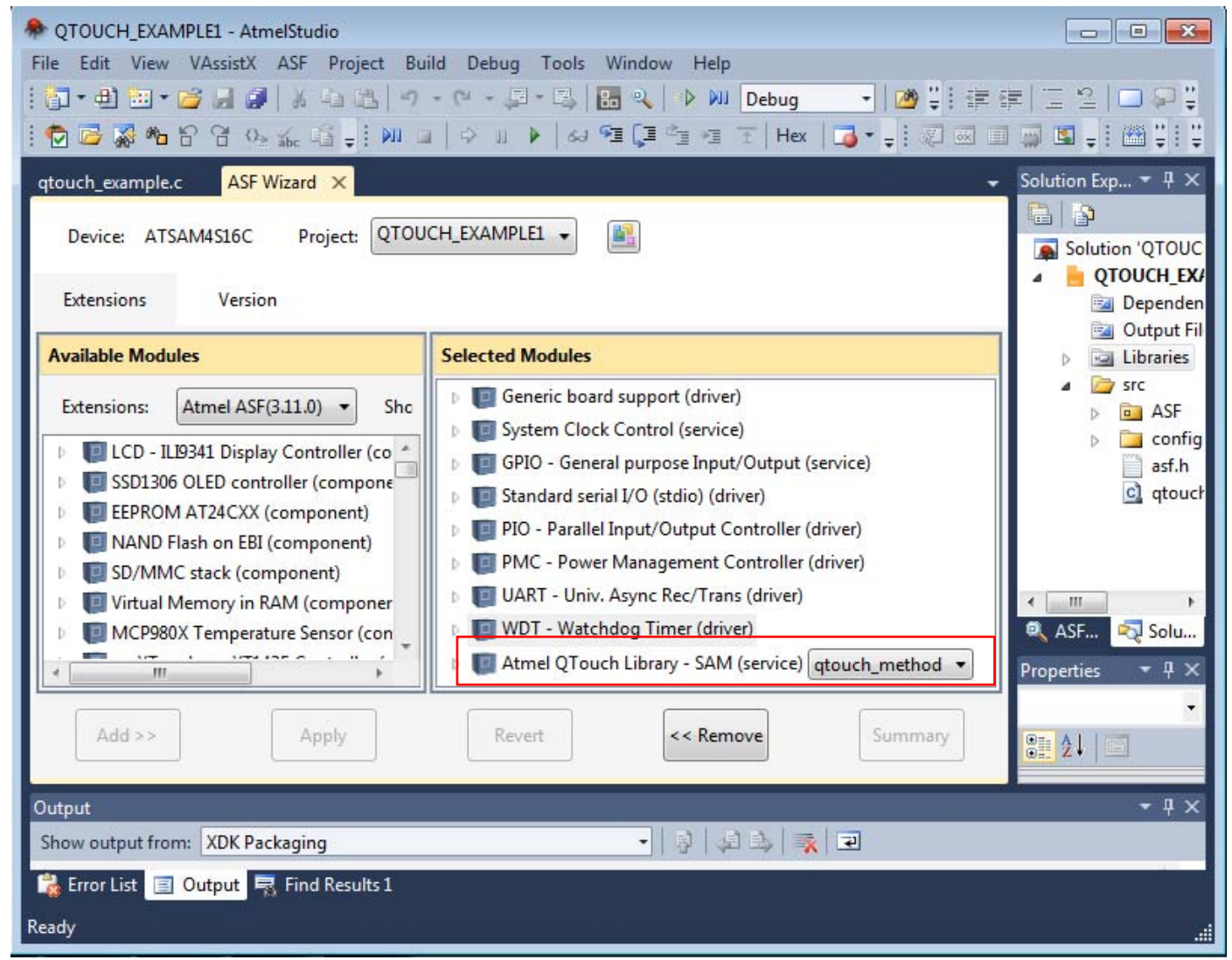

Figure 10 QTouch application development using ASF Wizard 


\section{Capstone Projects}

We have integrated the QTouch material into an advanced level MCU programming course for one year. Here, we reported two capstone projects designed by students. Touch devices helped improve the user interface of these projects, and made users operation more flexible and easy.

\section{Cyber Adaptive Spinny Thing}

This project presented a method to drive and control a Hi-Tec HS-311 servomotor using Atmel ARM Cortex M4. The speed of the servomotor is controlled by the QTouch Slider on the SAM4S-EK board and the direction is controlled by the QTouch buttons (RIGHT, LEFT and VALID) on board. The left button changes the direction to counter-clockwise, the right button changes the motor's direction to clockwise, and the middle button is used to start or stop the system. The graphic LCD screen of the microcontroller is also applied to display information such as the directional buttons as well as the slider. The slider is represented as a rectangle that corresponds to the duty cycle of the servomotor which will be displayed at the bottom of the screen. Figure 11(a) shows the wiring diagram, (b) displays the test setup and GUI. Students showed high interests in using QTouch to flexibly and smoothly control the operation of motor.
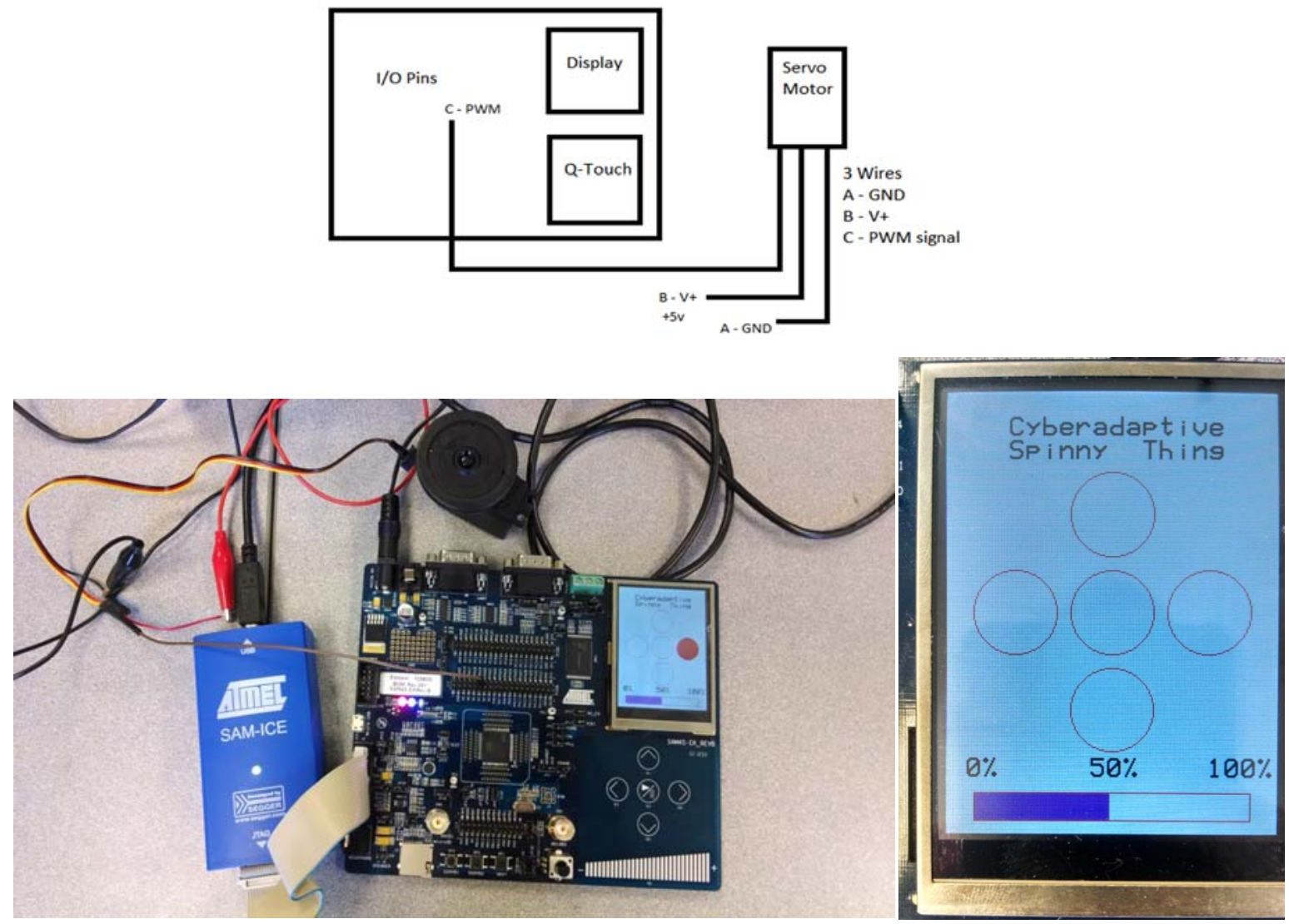

Figure 11 Capstone project (a) Design diagram, (b) Test setup, (c) GUI 


\section{Drawing on Touchable Graphical LCD}

This project is about drawing on the LCD screen display using the LCD screen touch and other QTouch elements on the SAM4S-EK board. It has four different drawing options. The UP and DOWN keys are programed to navigate through the options. The LEFT and RIGHT keys are for selecting to draw a square or a circle under each option. The Slider is responsible for changing the sizes of draws, for example, if users slide from left to right, the circles increase their radius. If users slide from right to lift, the radius of circles decreases. The option 3 and 4 allows users to draw circles or squares by touching the LCD resistive touch screen. The radius of drawing is computed by the touch position on the slider, the position of each drawing is at the touched position on the LCD.

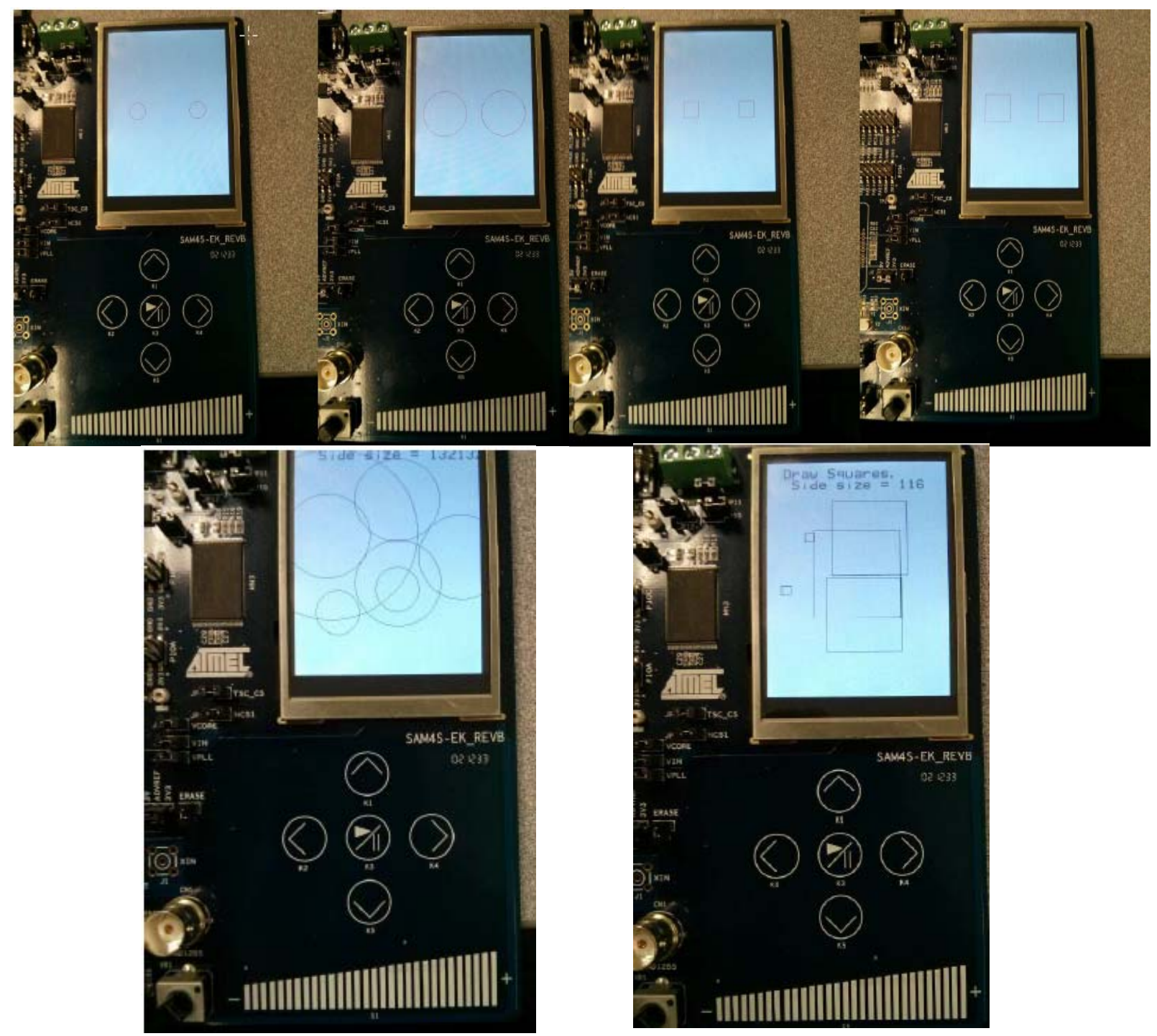

Figure 12 Four options of drawing on the touchable LCD

(a) Two circles sample draw (b) Two squares sample draw (c) Draw circles (d) Draw squares 
In these two projects, APIs provided by ASF library are used to draw rectangles and circles on LCD panel, and data structures and functions in QTouch library are used to read sensor data from QTouch components. Major API functions used are listed in the following table. The documentation of the APIs is also available online.

\begin{tabular}{|l|l|}
\hline Function/Data Structure Name & Use \\
\hline void qt_enable_slider(...) & Configures a set of channels as a slider \\
\hline void qt_enable_key(...) & Configures a channel as a touch key \\
\hline $\begin{array}{l}\text { struct }\{ \\
\text { uint8_t sensor_states[QT_NUM_SENSOR], } \\
\text { uint8_t rotor_slider_values[QT_MAX_Sliders] } \\
\text { \} qt_touch_status_t; }\end{array}$ & $\begin{array}{l}\text { Holds the status(on/off) of the touch key } \\
\text { sensors, and linear and angular positions of } \\
\text { sliders respectively. }\end{array}$ \\
\hline void ili9325_set_foreground_color $($ col $)$ & Sets foreground color \\
\hline uint32_t ili9325_draw_circle $(x, y, r)$ & Draws a circle of radius $r$ at $(\mathrm{x}, \mathrm{y})$ \\
\hline uint32_t ili9325_draw_filled_circle $(x, y, r)$ & Draws a filed circle of radius $r$ at $(\mathrm{x}, \mathrm{y})$ \\
\hline void ili9325_draw_line $(x 1, y 1, x 2, y 2)$ & $\begin{array}{l}\text { Draws a line, }(\mathrm{x} 1, \mathrm{y} 1) \text { is start coordinate, }(\mathrm{x} 2, \\
\mathrm{y} 2) \text { is end coordinate. }\end{array}$ \\
\hline void ili9325_draw_filled_rectangle $(x 1, y 1, x 2, y 2)$ & $\begin{array}{l}\text { Draws a filled rectangular, }(\mathrm{x} 1, \mathrm{y} 1) \text { is top-left } \\
\text { coordinates, }(\mathrm{x} 2, \mathrm{y} 2) \text { is bottom-right coordinate. }\end{array}$ \\
\hline
\end{tabular}

Table 2. Common API functions

\section{Conclusions}

This paper presents our experiences of teaching engineering and technology students the advanced Touch sensing technologies. More and more low-cost MCU development kits embedded with Touch elements become available for teaching Touch technologies. The Atmel SAM4S-EK board was employed in the course for students to conduct MCU programming projects. This evaluation board is equipped with rich resistive and capacitive Touch sensing elements. By utilizing the Atmel QTouch library and Atmel Software Framework, students are able to develop MCU-based capstone projects using Touch devices in various ways at the end of the course. Some enhanced the user interface of traditional motor control projects; some are Touch centered projects for multi-media applications. In the future, we will encourage students to develop more complex MCU projects using Touch devices.

\section{Bibliography}

1. White paper - "Comparison All Resistive Touch Technologies", 2014.

http://www.elotouch.com/Technologies/compare_resist.asp 
2. Touch Technology Brief from 3M Touch Systems, "Projected Capacitive Technology", 2013.

3. Geoff Walker, "Fundamentals of Touch Technologies", 2013. www.walkermobile.com/Touch_Technologies_Tutorial_Latest_Version.pdf

4. Atmel QTouch Library: http://www.atmel.com/products/TouchSolutions/touchsoftware/default.aspx 